\title{
Stability and superconductivity of K-Y-H hydrides under high pressure
}

\author{
Ling-Yan Chen, Wen-Cai Lu, Hai-Liang Chen, Bing-Yu Li, Kai-Ping Yang \\ College of Physics and State Key Laboratory of Bilological Polysaccharide Fiber Forming and Ecological Textile, \\ Qingdao University, Qingdao, Shandong 266071, PR China \\ Institute of Theoretical Chemistry, Jilin University, Jilin 130021, P. R. China
}

\begin{abstract}
It has been a hot issue to search for hydrides at high pressure with high superconducting transition temperature $(\mathrm{Tc})$. In this work, ternary hydrogen-rich compounds KYHn ( $=1-16)$ were studied and calculated using the density function theory (DFT) method and he results showed that the $\mathrm{KYH}_{8}$ demonstrates good stability and superconductivity. And keep thermodynamically stable in the pressure range of 50$300 \mathrm{GPa}$. The research found that the $\mathrm{Tc}$ of $\mathrm{KYH}_{8}-\mathrm{C} 2 / \mathrm{m}$ was predicted to be $111.3 \mathrm{~K}$, $122.0 \mathrm{~K}$, and $142.3 \mathrm{~K}$ at 200,250 and $300 \mathrm{GPa}$, respectively. Our current study provides a possibility for searching new high-Tc superconductors in ternary hydrides.
\end{abstract}

\section{Introduction}

The search for high-temperature superconductors is still an exciting subject, as its wide application has aroused great interest among scientists. Based on the BCS electron-phonon superconducting theory, high frequency phonons, strong electronphonon interactions, and large electron state density (DOS) near the Fermi level contribute to the high superconducting transition temperature Tc. Hydrogen is the lightest element in nature and promotes high phonon frequency and electron-phonon coupling. Theoretical predictions show that when solid hydrogen turns to metal at high pressure, they are very likely to become high temperature superconductors. The concept of metal superconductivity has been extended to hydride-rich compounds. In 2004, Ashcroft [5] predicted that "chemical precompression" could be caused by heavier elements, metallize hydrogen-rich materials at much lower pressures than pure hydrogen. Theoretical and experimental results show that many hydrogen-rich compounds, such as $\mathrm{H}_{3} \mathrm{~S}[10], \mathrm{H}_{2} \mathrm{~S}, \quad \mathrm{LaH}_{10}$ [18], $\mathrm{YH}_{10}, \mathrm{YH}_{6}$, [27] $\mathrm{CaH}_{6}$, [29] 
$\mathrm{MgH}_{6}$,exhibit high Tc under high pressure. Theoretical research predicts that when $\mathrm{H}_{3} \mathrm{~S}$ is in 200GPa, Tc can reach 191-204K[10] with Im-3m phase. And the prediction was confirmed in subsequent experiments. For the alkali metal compounds, CaH6 with a cage structure at a high pressure had a predict Tc of $235 \mathrm{~K}$ at $150 \mathrm{GPa}, \mathrm{MgH} 6$ critical temperature of $\sim 260 \mathrm{~K}$ above $300 \mathrm{GPa}$ and KH6[8] superconducting transition temperature in the range of $58.6 \mathrm{~K}$ to $\sim 69.8 \mathrm{~K}$ at $166 \mathrm{GPa}$. In addition, the theoretically predicted that $\mathrm{YH}_{3}$ is most stable in thermodynamics, has a Tc of $40 \mathrm{~K}$ at $17.7 \mathrm{GPa}[3]$, YH6 and YH10 ,the $\mathrm{H}$ atoms form a sodalite-like cage and $\mathrm{H}_{32}$ cage[13], respectively, have critical temperature of $251-264 \mathrm{~K}$ at $120 \mathrm{GPa}$ and $305-326 \mathrm{~K}$ at $250 \mathrm{GPa}$. $\mathrm{LaH}_{10}$ had Tc of $274-286 \mathrm{~K}$ at $210 \mathrm{GPa}$.

Recently, ternary hydrides have been studied. The superconducting properties of several kinds of ternary hydrides have been reported. At 100GPa, $\mathrm{BaReH}_{9}[11]$ has been reported to be a superconductor with a Tc of $\sim 7 \mathrm{~K}$. In 2017, $\mathrm{MgGeH}_{6}[12]$ was reported to be a potential high temperature superconductor with a Tc of $\sim 67 \mathrm{~K}$ at $200 \mathrm{GPa}$. In the near future, theoretical research reveals that $\mathrm{CaYH}_{12}$ becomes stable with a cubic Fd3m structure above $170 \mathrm{GPa}$, with Tc of $\sim 258 \mathrm{~K}$ at $200 \mathrm{GPa}$ [28]. Then, calculation shows that $\mathrm{KScH}_{12}$ with a hydrogen cage has critical temperature of $122 \mathrm{~K}$ at $300 \mathrm{GPa}$, and $\mathrm{GaAsH}_{6}$ with a Tc of $\sim 98 \mathrm{~K}$ at $180 \mathrm{GPa}$.

In this paper, we studied the ternary hydrides of $\mathrm{KYH}_{\mathrm{n}}$ at a pressure range of 100 to 300 GPa. We used genetic algorithm (GA) to search for the structure of KYHn and then studied their thermodynamic stabilities, electronic properties and superconductivity. The most stable structure of $\mathrm{KYH}_{8}$ is cubic $\mathrm{c} 2 / \mathrm{m}$, it was predicted $\mathrm{Tc}$ to be $0.3 \mathrm{~K}$ at $50 \mathrm{GPa}, 111 \mathrm{~K}$ at $200 \mathrm{GPa}$, and $141 \mathrm{~K}$ at $300 \mathrm{GPa}$.

\section{2. computation details}

The low-enthalpy structures of KYHn $(n=1-16)$ at high pressure were searched by genetic algorithm (GA) combined with structural optimizations using density functional theory (DFT). We used the Perdew-Burke-Ernzerhof (PBE) Generalized gradient approximation (GGA) [4] functional and Norm-conserving pseudopotentials, implemented in the CASTEP code are employed. The GA search of low-enthalpy structures of KYHn were performed at $100 \mathrm{GPa}$. In the GA search, a coarse 
optimization method was used with an energy cutoff of $330 \mathrm{eV}$ and a k-mesh of $5 \times 6$ $\times 7$. Then the structures from the GA search were re-optimized with high precision at the pressure range 50 - $300 \mathrm{GPa}$, with energy cutoff of $1000 \mathrm{eV}$ and a k-point mesh of $2 \pi \times 0.03 \AA-1$ to determine low-enthalpy structures. The Quantum-ESPRESSO package [6] was used to calculate phonon, electron-phonon coupling (EPC) and Tc, using the PBE functional, Norm-conserving pseudopotential and an energy cutoff of 70 Ry.

\section{Results and discussion}

\subsection{Structural stabilityof K-Y-H}

The stable structures of KYHn were studied at the pressure range of 100-300 GPa. Unlike binary compounds, the synthesis and decomposition pathways of ternary compounds are more complicated.

(a)

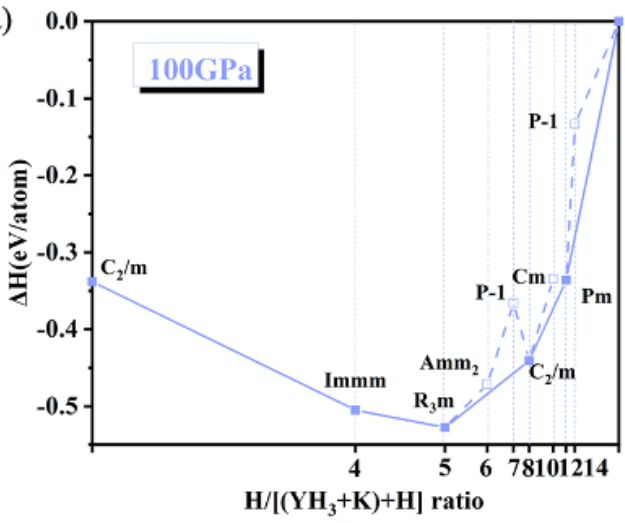

(b)

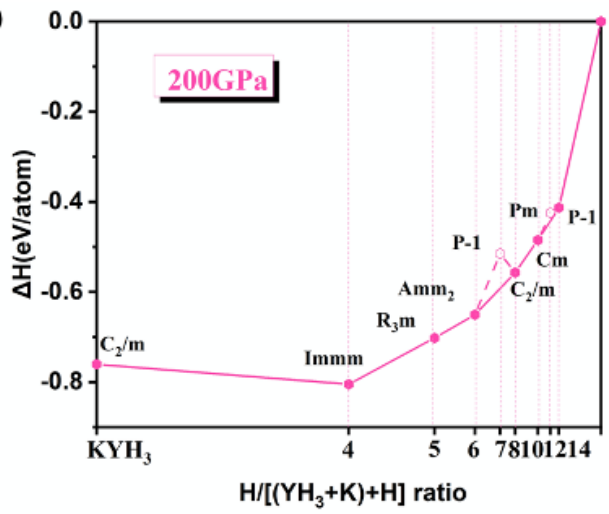

(c)

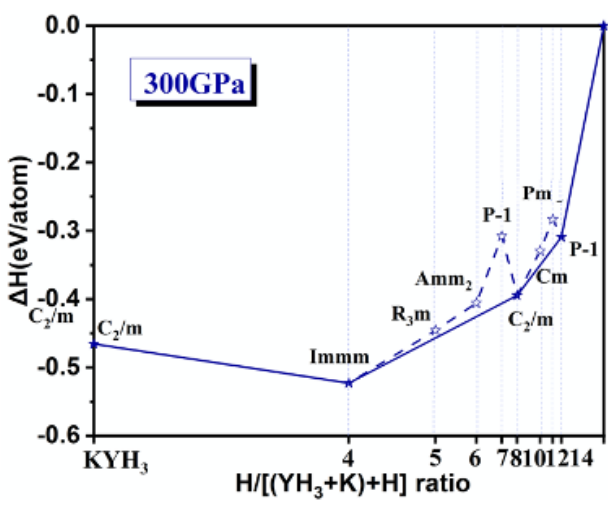

Figure 1 Calculated formation enthalpies $(\Delta \mathrm{H})$ of various K-Y-H stoichiometries at 100, 200 and 300 GPa with respect to $(\mathrm{K}+\mathrm{YH} 3)+1 / 2$ solid $\mathrm{H} 2$. The solid lines represent convex hull and solid symbols on convex hull indicate stable stoichiometries. Hollow symbols connect by dashed lines represent unstable stoichiometries 
The calculated formation enthalpies of the KYHn hydrides at 100, 200 and 300 GPa with respect to $\left(\mathrm{K}+\mathrm{YH}_{3}\right)+1 / 2$ solid $\mathrm{H}_{2}$. It can be seen that $\mathrm{KYH}_{5}$ is most stable at $100 \mathrm{GPa}$, and $\mathrm{KYH}_{4}$ is most stable at $200-300 \mathrm{GPa}$.The $\mathrm{KYH} 3,4,5,8.12$ at $100 \mathrm{GPa}$, $\mathrm{KYH} 3,4,5,6,8,10$ and 12 at $200 \mathrm{GPa}, \mathrm{KYH} 3,4,8$ and 14 at $300 \mathrm{GPa}$ locate on the convex hull meaning they may be thermodynamically stable (Figure 1). We extend the structure to 2 f.u. Among them, the crystal structures of $\mathrm{KYH} 8-\mathrm{C} 2 / \mathrm{m}$ and $\mathrm{KYH}_{14}-\mathrm{P}-1$ at $300 \mathrm{GPa}$ were shown in Figure 2. In the figure2, $\mathrm{KYH}_{8}-\mathrm{C} 2 / \mathrm{m}$ and $\mathrm{KYH}_{14}-\mathrm{P}-1$ in the directions of $001,010,100$ are shown, in which the pink atom is $\mathrm{H}$, the yellow atom is $\mathrm{K}$, and the blue atom is $\mathrm{Y}$. For $\mathrm{KYH}_{8}$, we can observe the presence of the $\mathrm{H}_{3}$ unit in the structure and the heavy atom Y combined with two hydrogen to form a straight chain structure, in which the $\mathrm{H}_{3}$ unit is likely to be the source of high temperature superconductivity. There are two types of $\mathrm{H}-\mathrm{H}$ bonds in the crystal, with distances of $0.921 \AA(\mathrm{H} 1-\mathrm{H} 2)$, $0.819 \AA\left(\mathrm{H}_{3}-\mathrm{H}_{5}\right)$, and the bond length between $\mathrm{H}_{7}$ and $\mathrm{Y}$ is $1.77338 \AA$ (Figure 2). Their detaileds were given in Table S2 in the supplementary material.

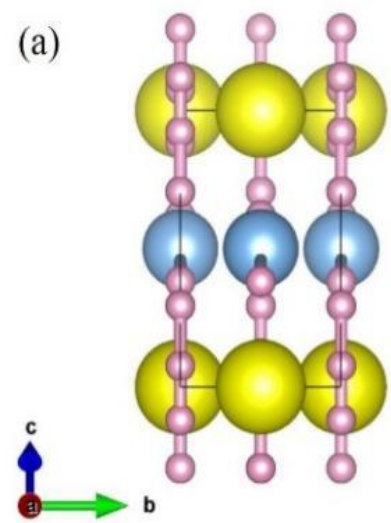

(c)

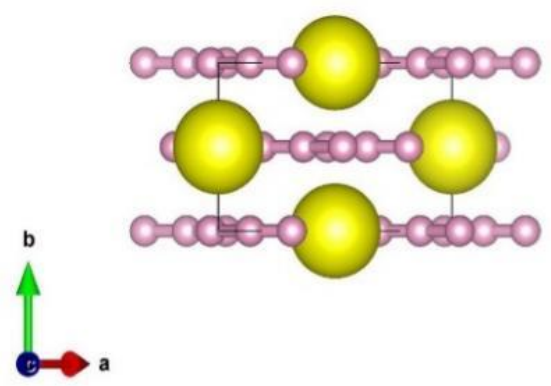

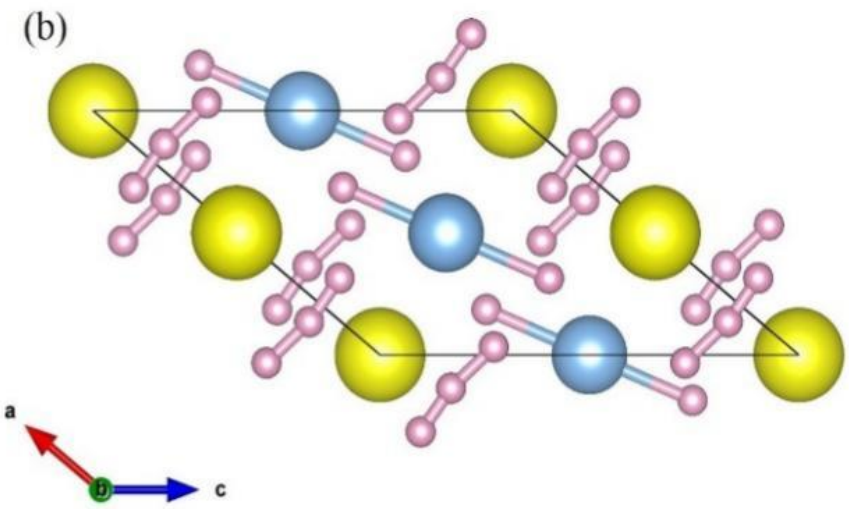

(d)

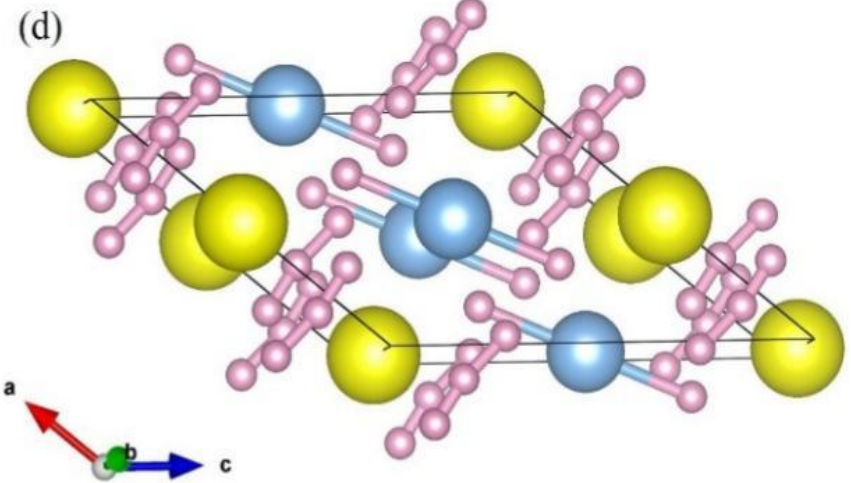

We caculation the electronic localization function of $\mathrm{C} 2 / \mathrm{m} \mathrm{KYH}_{8}$ at $300 \mathrm{GPa}$ to explore the bonding information. The ELF has maps values in the range from 0 to 1 , 
with 1 corresponding to excellent localization of valence electrons, standing for a strong covalent bond. The ELF values for the $\mathrm{H}_{1}-\mathrm{H}_{3}$ and $\mathrm{H}_{3}-\mathrm{H}_{5}$ bonds are nearly 0.9 , meaning they are strong covalent bonds. The ELF value of the $\mathrm{Y}-\mathrm{H}_{7}$ bond in the octagon is approximately 0.5 , which is the corresponding value for the homogeneous electron gas (Fig. 3(a)). Fig. 4 shows the calculated relative enthalpy curves for the predicted structures of $\mathrm{KYH}_{8}$ as a function of pressure. The enthalpies of the decomposition to binary hydrides were also taken into account. On the basis of corresponding binary compounds, the decomposition enthalpies into $\mathrm{K}+\mathrm{Y}+4 \mathrm{H}_{2}, \mathrm{~K}+\mathrm{YH} 3+5 / 2 \mathrm{H}_{2}, \mathrm{~K}+\mathrm{YH}_{6}+\mathrm{H}_{2}$, and $\mathrm{KH}_{6}+\mathrm{Y}+\mathrm{H}_{2}$ are considered in order to investigate the stability of KYH8. For KYH8,
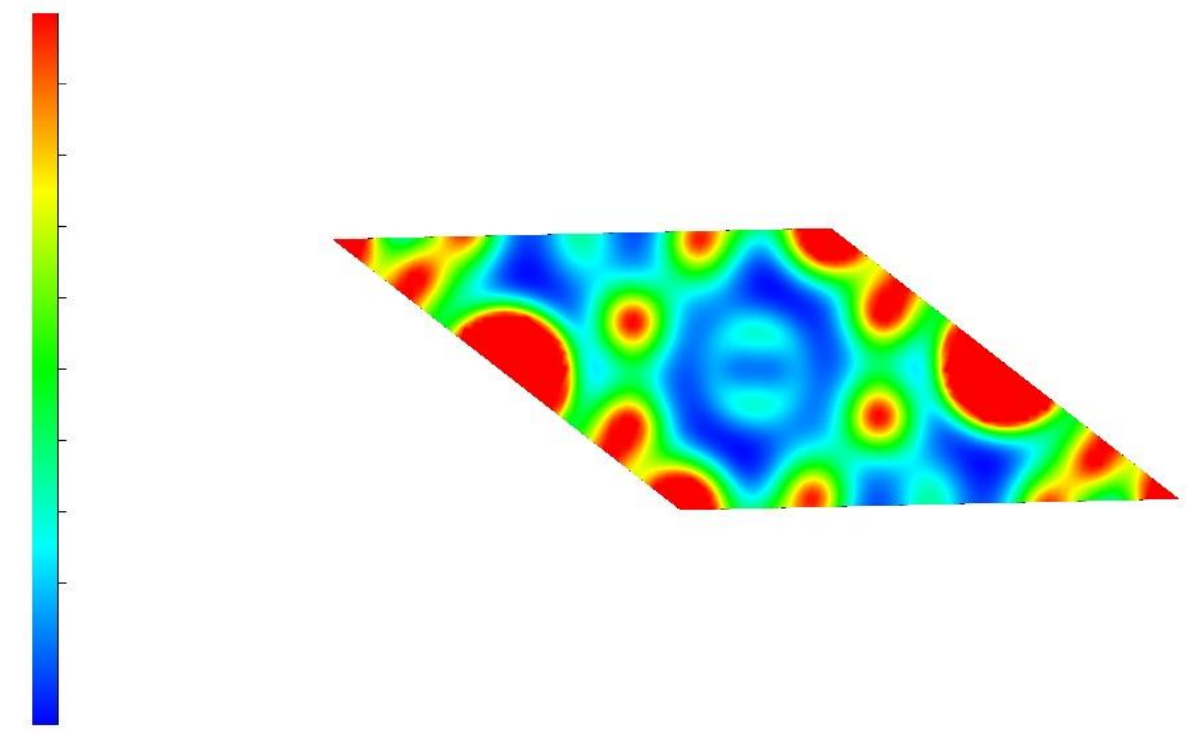

Figure 3 The electronic localization function of $\mathrm{C} 2 / \mathrm{m} \mathrm{KYH} 8$

four energetically competing phases $\mathrm{C} 2 / \mathrm{m}, \mathrm{Pmm} 2, \mathrm{Amm} 2$, and $\mathrm{Cm}$ were found by our structural predictions. The crystal structures and lattice parameters are shown in Fig. S3. Without considering zero-point energy (ZPE), we found that the $\mathrm{c} 2 / \mathrm{m}$ is the most stable phase below 90GPa. Above 93GPa, the phase of Amm2 becomes thermodynamically stable above $90 \mathrm{GPa}$. The results can show $\mathrm{KYH}_{8}$ is stable with respect to $\mathrm{K}+\mathrm{YH}_{3}+5 / 2 \mathrm{H}_{2}$ in the pressure range, and can still table with respect to $\mathrm{KH}_{6}+\mathrm{Y}+\mathrm{H}_{2}$ below $280 \mathrm{GPa}$.

3.2Electronic properties, dynamic Properties and superconductivity of $\mathrm{KYH}$ 


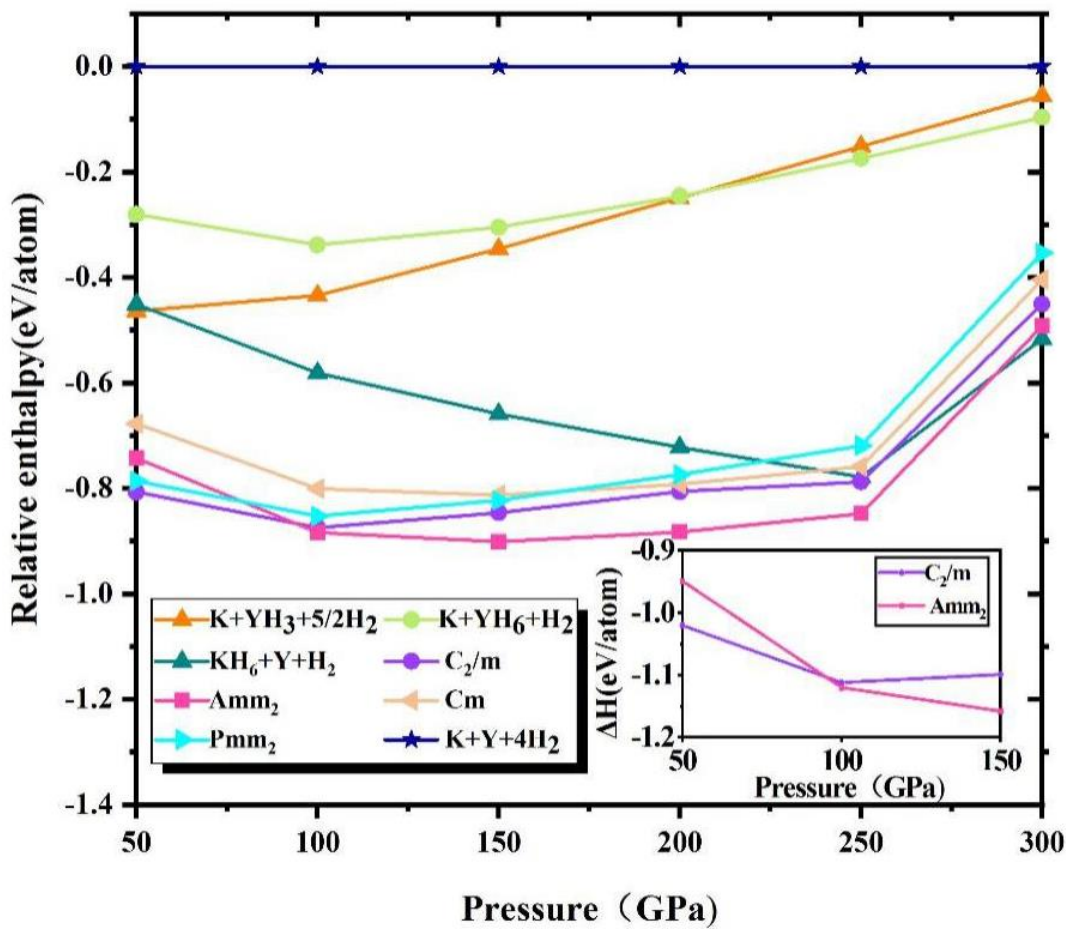

Figure 4 The calculated enthalpies of various structures for (a) $\mathrm{KYH}_{8}$ as a function of pressure with respect to the $\mathrm{K}+\mathrm{Y}+4 \mathrm{H}_{2}$. The decomposition enthalpies for $\mathrm{KYH}_{8}$ to $\mathrm{K}+\mathrm{YH} 3+5 / 2 \mathrm{H}_{2}, \mathrm{~K}+\mathrm{YH}_{6}+\mathrm{H}_{2}, \mathrm{KH}_{6}+\mathrm{Y}+\mathrm{H}_{2}$ structure including zeropoint corrections as a function of pressure.

(a)

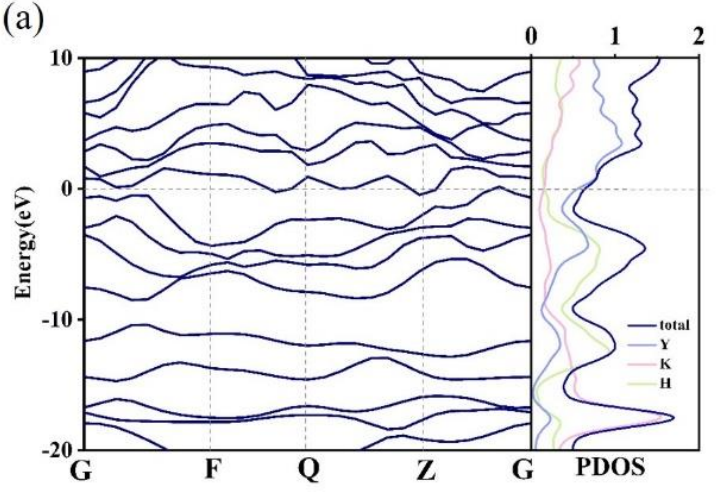

(b)

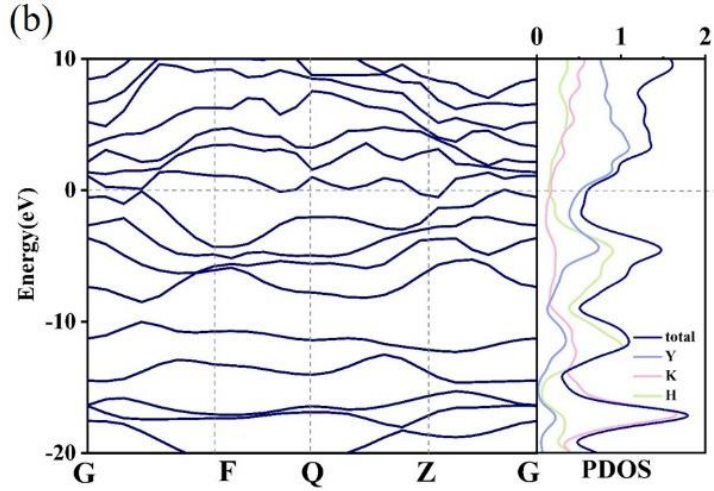

(c)

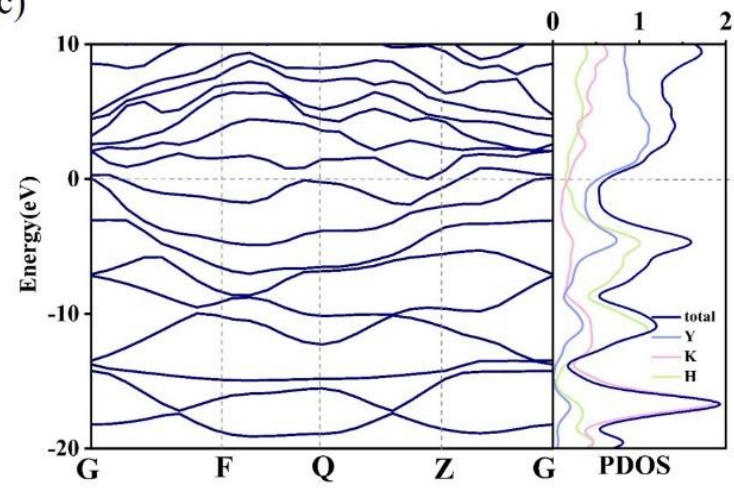

Figure 5 Calculated electronic band structures and partial density of states (PDOSs) of (a) $\mathrm{KYH}_{8}-\mathrm{c} 2 / \mathrm{m}$ at $200 \mathrm{GPa}$,

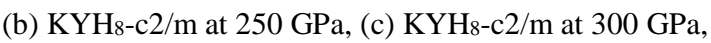




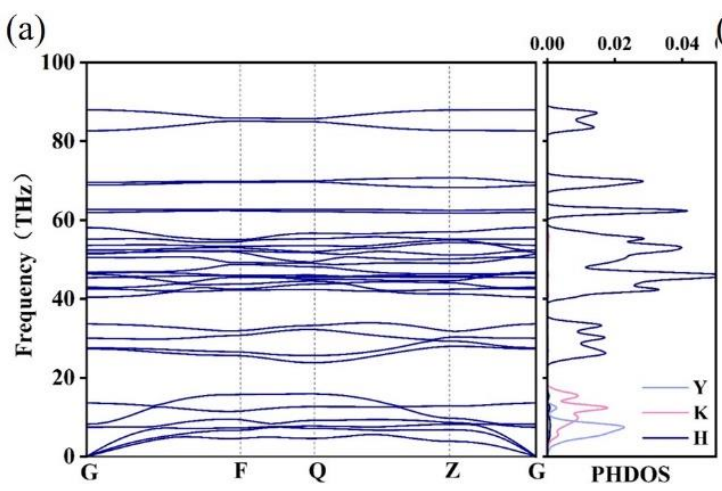

(b)
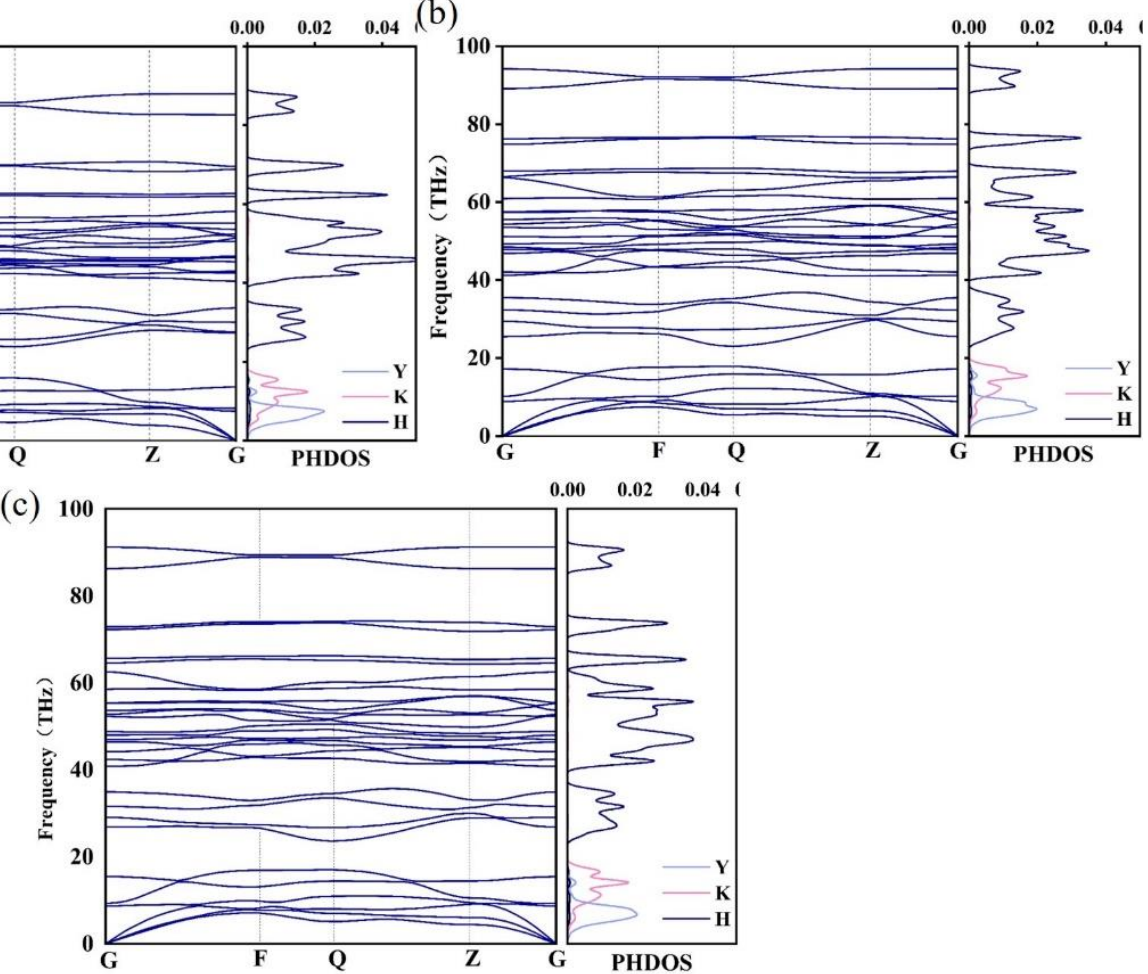

Figure 4 Calculated phonon dispersion curves, phonon density of states (PHDOSs) of $\mathrm{KYH}_{8}-\mathrm{C} 2 / \mathrm{m}$ (a) KYH8-c2/m at $200 \mathrm{GPa}$, (b) KYH8-c2/m at $250 \mathrm{GPa}$, (c) KYH8-c2/m at $300 \mathrm{GPa}$,

For $\mathrm{KYH}_{8}$, we further research their electronic dynamic and their superconducting properties. In order to confirm whether they are metals under the pressure, we analyzed the electronic band structures and projected density of states (PDOS) for the predicted structures. As shown in Figure 4 (a), (b)and (c), There are many bands crossing the Fermi level, the band gaps of KYH8-c2/m at 200, 250 and 300GPa are zero, indicating they are metallic. the overlap of the valence band and the conduction band in the Fermi level reflects the metallic properties of the structure. The energy band on the Fermi level can effectively affect the critical temperature. There are some flat bands around the Fermi level, which may contribute to enhance electron-phonon interactions. To explore the electronic, dynamic and mechanical properties of KYH8, we calculated the phonon dispersion curves, phonon density of states (PHDOS) of KYH8-C2/m and. The results indicate that there phonon dispersion curves has no virtual frequency (Figure5), which can help us determine their structures are dynamic stable. As shown in the phonon DOS, the vibrations of the heavier $\mathrm{Y}$ and $\mathrm{K}$ atoms are contributed to the lowfrequency phonon branches, while vibrations of $\mathrm{H}$ atoms are associated with the high frequency regions, respectively. Based on the stability of the structure at various 
pressures, we calculated Eliashberg spectral function $\alpha^{2} \mathrm{~F}(\omega)$ and electron phonon coupling (EPC) parameters $\lambda(\omega)$ of $\mathrm{KYH}_{8}-\mathrm{c} 2 / \mathrm{m}$ to further explore the superconducting properties. There are two parameters that can be used to estimate $\mathrm{Tc}\left(\mathrm{T}_{\mathrm{c}}=\right.$ $\left.\frac{\omega_{\log }}{1.2} \exp \left[-\frac{1.04(1+\lambda)}{\lambda-\mu^{*}(1+0.62 \lambda)}\right]\right)$, calculating electron phonon coupling (EPC) parameter $\lambda$ and the logarithmic average phonon frequency wlog, and Eliashberg phonon spectral function $\alpha^{2} F(\omega)$ and $\lambda$ are shown in Fig. 6
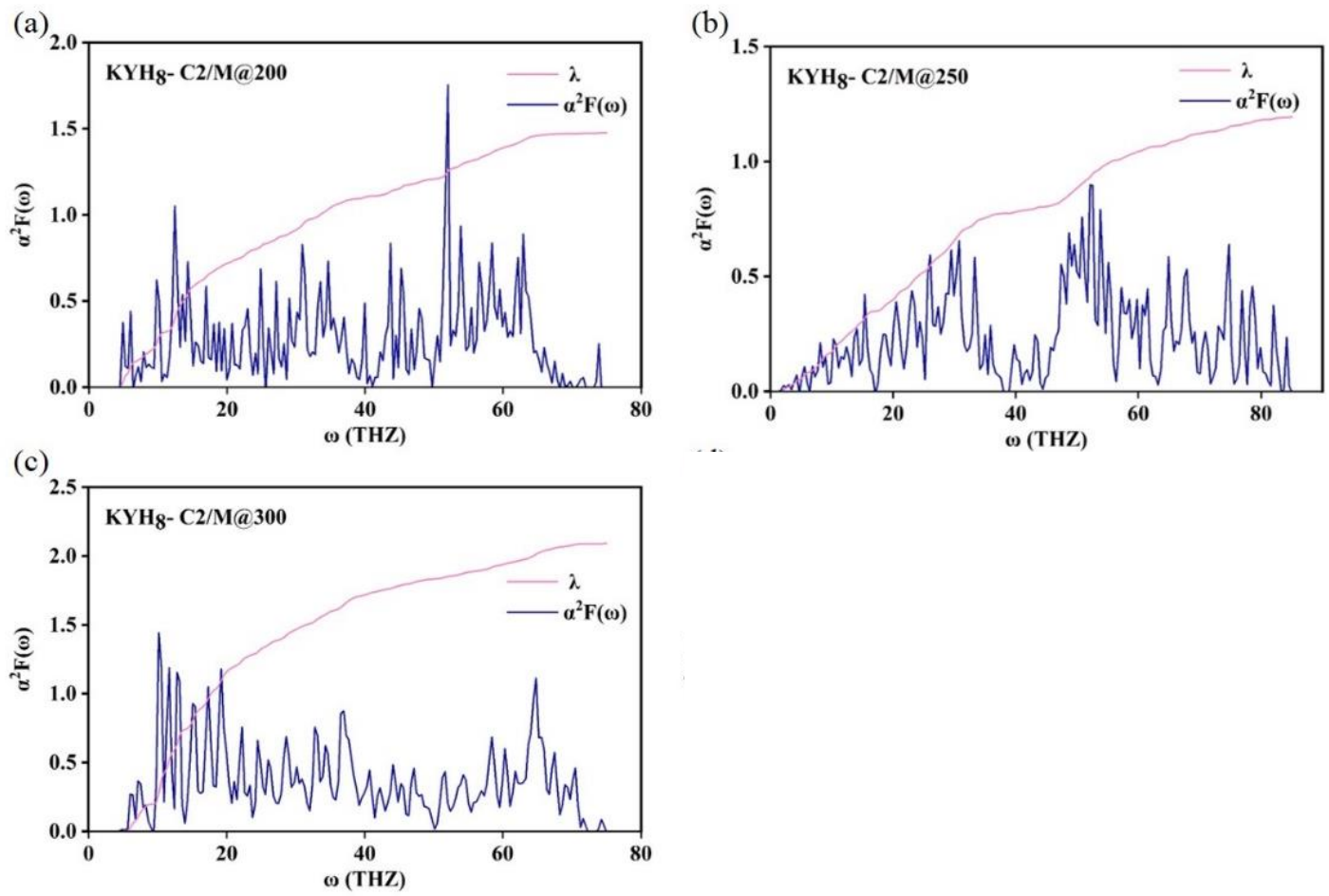

Figure 6 Eliashberg phonon spectral function $\alpha^{2} \mathrm{~F}(\omega)$, and electron-phonon coupling parameter $\lambda$ of (a) $\mathrm{KYH}_{8}-\mathrm{c} 2 / \mathrm{m}$ at $200 \mathrm{GPa}$, (b) $\mathrm{KYH}_{8}-\mathrm{c} 2 / \mathrm{m}$ at $250 \mathrm{GPa}$, (c) $\mathrm{KYH} 8-\mathrm{c} 2 / \mathrm{m}$ at $300 \mathrm{GPa}$,

Table 1 Calculated EPC parameter $\lambda, \omega \log$, DOS at the Fermi level N(cf), and Tc at different pressures.

\begin{tabular}{lllllll}
\hline & $\begin{array}{l}\text { Pressure } \\
(\mathrm{GPa})\end{array}$ & $\lambda$ & $\omega_{\log }(\mathrm{K})$ & $\mathrm{N}\left(\mathrm{E}_{\mathrm{f}}\right)$ & $\begin{array}{l}\mathrm{Tc}(\mathrm{K}) \\
\mu^{*}=0.1\end{array}$ & $\begin{array}{l}\mathrm{Tc}(\mathrm{K}) \\
\mu^{*}=0.13\end{array}$ \\
& 200 & 1.478 & 989.897 & 2.76 & 111.3 & 101.4 \\
$\mathrm{C} 2 / \mathrm{m}-\mathrm{KYH}_{8}$ & 250 & 1.214 & 1342.505 & 4.47 & 122.0 & 108.3 \\
& 300 & 2.09 & 960.470 & 0.65 & 142.3 & 133.2 \\
\hline
\end{tabular}

And then, we calculated the electron-phonon coupling (EPC) parameter $\lambda$, the phonon frequency logarithmic average $\left(\omega_{\log }\right)$ and DOS at the Fermi level $\mathrm{N}_{(\varepsilon f)}$ by QE software package. We chose the Coulomb pseudopotential $\mu *$ of 0.1 and 0.3 , the 
estimated Tc values for $\mathrm{C} 2 / \mathrm{m}-\mathrm{KYH}_{8}$ are $111.3 \mathrm{~K}, 101.4 \mathrm{~K}, 142.3 \mathrm{~K}$ and $122.0 \mathrm{~K}, 108.3 \mathrm{~K}$, $133.2 \mathrm{~K}$ at 200,250 and $300 \mathrm{GPa}$, respectively (Table 1). It is obvious that the transition temperature increases with pressure.

\section{Conclusions}

In summary, the crystal structures and stability of the KYHn under high pressure were searched by GA and combined with the structural optimizations with DFT. And we systematically studied the structures and stabilities of KYHn at the pressure range 50-300 GPa. It is found that KYH8 c2/m have strong electron phonon coupling and a high Tc $(142.3 \mathrm{~K}$ and $152.3 \mathrm{~K}$ at $300 \mathrm{GPa})$. The $\mathrm{H} 3$ unit is found in the structure of $\mathrm{KYH}_{8}$ c $2 / \mathrm{m}$ likely to lead to a higher Tc.

\section{Declaration of competing interest}

The authors declare that they have no known competing financial interests or personal relationships that could have appeared to influence the work reported in this paper.

\section{Acknowledgments}

This work was supported by the National Natural Science Foundation of China (Grant No. 21773132).

\section{References}

[1] P. Hohenberg, W. Kohn, Inhomogeneous Electron Gas. Physical Review 136, B864-B871 (1964).

[2] W. Kohn, L. J. Sham, Self-Consistent Equations Including Exchange and Correlation Effects. Physical Review 140, A1133-A1138 (1965).

[3] Y. Wang, M. Y. Chou, Structural and electronic properties of hexagonal yttrium trihydride. Phys Rev B Condens Matter 51, 7500-7507 (1995)

[4] J. P. Perdew, K. Burke, M. Ernzerhof, Generalized Gradient Approximation Made Simple. Physical Review Letters 77, 3865-3868 (1996).

[5] N. W. Ashcroft, Hydrogen Dominant Metallic Alloys: High Temperature Superconductors? Physical Review Letters 92, 187002 (2004).

[6] P. Giannozzi et al., QUANTUM ESPRESSO: a modular and open-source software project for quantum simulations of materials. Journal of Physics: Condensed Matter 21, 395502 (2009).

[7] J. Lv, Y. Wang, L. Zhu, Y. Ma, Predicted novel high-pressure phases of lithium. Phys Rev Lett 106, 015503 (2011).

[8] D. Zhou et al., Ab initiostudy revealing a layered structure in hydrogen-rich KH6under high pressure. Physical Review B 86, (2012).

[9] A. O. Lyakhov, A. R. Oganov, H. T. Stokes, Q. Zhu, New developments in evolutionary structure prediction algorithm USPEX. Computer Physics Communications 184, 1172-1182 (2013). 
[10] D. Duan et al., Pressure-induced metallization of dense (H2S)2H2 with high-Tc superconductivity. Scientific Reports 4, 6968 (2014).

[11] T. Muramatsu et al., Metallization and Superconductivity in the Hydrogen-Rich Ionic Salt BaReH9. The Journal of Physical Chemistry C 119, 18007-18013 (2015).

[12] Y. Ma et al., Prediction of superconducting ternary hydride MgGeH6: from divergent high-pressure formation routes. Phys Chem Chem Phys 19, 27406-27412 (2017).

[13] T. Ishikawa, T. Miyake, K. Shimizu, Materials informatics based on evolutionary algorithms: Application to search for superconducting hydrogen compounds. Physical Review B 100, (2019).

[14] X. Liang et al., First-principles study of crystal structures and superconductivity of ternary \$ $\{\backslash \text { mathrm }\{\mathrm{YSH}\}\}_{-}\{6\} \$$ and $\$\{\backslash \mathrm{mathrm}\{\operatorname{LaSH}\}\}_{-}\{6\} \$$ at high pressures. Physical Review B 100, 184502 (2019).

[15] Z. Shao et al., Ternary superconducting cophosphorus hydrides stabilized via lithium. npj Computational Materials 5, (2019).

[16] Y. Sun, J. Lv, Y. Xie, H. Liu, Y. Ma, Route to a Superconducting Phase above Room Temperature in ElectronDoped Hydride Compounds under High Pressure. Physical Review Letters 123, 097001 (2019).

[17] S. Di Cataldo, W. von der Linden, L. Boeri, Phase diagram and superconductivity of calcium borohyrides at extreme pressures. Physical Review B 102, (2020).

[18] M. Kostrzewa, K. M. Szczęśniak, A. P. Durajski, R. Szczęśniak, From LaH10 to room-temperature superconductors. Scientific Reports 10, 1592 (2020).

[19] D. V. Semenok, I. A. Kruglov, I. A. Savkin, A. G. Kvashnin, A. R. Oganov, On Distribution of Superconductivity in Metal Hydrides. Current Opinion in Solid State and Materials Science 24, (2020).

[20] A. M. Shipley, M. J. Hutcheon, M. S. Johnson, R. J. Needs, C. J. Pickard, Stability and superconductivity of lanthanum and yttrium decahydrides. Physical Review B 101, 224511 (2020).

[21] W. Sukmas, P. Tsuppayakorn-aek, U. Pinsook, T. Bovornratanaraks, Near-room-temperature superconductivity of $\mathrm{Mg} / \mathrm{Ca}$ substituted metal hexahydride under pressure. Journal of Alloys and Compounds 849, (2020).

[22] C. Wang, S. Yi, S. Liu, J.-H. Cho, Underlying mechanism of charge transfer in Li-doped $\$\{\backslash \text { mathrm }\{\mathrm{MgH}\}\}_{-}\{16\}$ at high pressure. Physical Review B 102, 184509 (2020).

[23] N. Zaremba et al., LaNiGe with Non-centrosymmetric LaPtSi Type Structure. Zeitschrift für anorganische und allgemeine Chemie, (2020).

[24] P. Zhang, Y. Sun, X. Li, J. Lv, H. Liu, Structure and superconductivity in compressed Li-Si-H compounds: Density functional theory calculations. Physical Review B 102, 184103 (2020).

[25] P. Tsuppayakorn-aek, W. Sukmas, R. Ahuja, W. Luo, T. Bovornratanaraks, Stabilization and electronic topological transition of hydrogen-rich metal Li5MoH11 under high pressures from first-principles predictions. Scientific Reports 11, 4079 (2021).

[26] Y. K. Wei et al., Formation and superconducting properties of predicted ternary hydride ScYH6 under pressures. International Journal of Quantum Chemistry 121, e26459 (2021).

[27] W.-H. Yang et al., Superconductivity in alkaline earth metal doped boron hydrides. Physica B: Condensed Matter 611, 412795 (2021).

[28] Liang X, Bergara A, Wang L, et al. Potential high- Tc superconductivity in CaYH12 under pressure[J]. Physical Review B, 2019, 99(10).

[29] Hui Wang, John S. Tse, Kaori Tanaka, Toshiaki Iitaka, Yanming Ma Proceedings of the National Academy of Sciences Apr 2012, 109 (17) 6463-6466; 